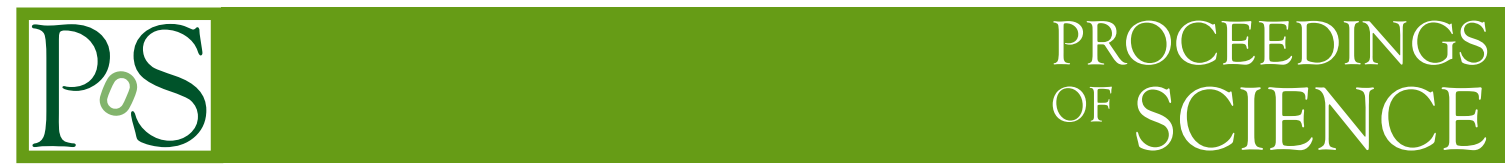

\title{
Top-quark mass measurements at the LHC: alternative methods
}

\author{
Marcel Vos*, on behalf of the ATLAS and CMS collaborations \\ IFIC (UVEG/CSIC) Valencia \\ Apartado de Correos 22085 \\ E-46071 Valencia - Spain \\ E-mail: Marcel.Vosdific.uv.es
}

\begin{abstract}
Alternative top quark mass determinations can provide inputs to the world average with orthogonal systematic uncertainties and may help to refine the interpretation of the standard method. Among a number of recent results I focus on the extractions by ATLAS and CMS of the top quark pole mass from the $t \bar{t}$ pair and $t \bar{t}+1$ jet production cross-section, which have now reached a precision of $1 \%$.
\end{abstract}

8th International Workshop on Top Quark Physics

14-18 September, 2015

Ischia, Italy

\footnotetext{
* Speaker.
} 


\section{Introduction}

The top quark mass is one of the key parameters of the Standard Model (SM). The SM predicts a relation between the top quark mass and other parameters of the theory (in particular the masses of the $\mathrm{W}$ and Higgs bosons). The top quark mass is the main input to extrapolations of the Higgs potential to high scales. A precise measurement of the top quark mass thus allows for a stringent test of the self-consistency of the theory. A precise measurement is furthermore needed to reduce the parametric uncertainties on many SM predictions.

Contrary to other quarks, the top quark decays before it hadronizes. One can therefore observe a mass peak directly by reconstructing the products of the decay chain $t \rightarrow W b \rightarrow q \bar{q} b \rightarrow$ jets of hadrons. As the partonic final state contains coloured objects, observables describing the final state objects receive corrections from the parton shower and non-perturbative effects. The most precise measurements of the top quark mass extract the mass by comparing distributions generated using Monte Carlo (MC) generators to the data. The mass parameter is usually identified with the pole mass. Such direct measurements of the top quark mass have attained a precision of better than $0.5 \%$ [1], already exceeding the pre-LHC expectations [2]. The current world average is dominated by systematic uncertainties on the response to jets of the experiments and in the modelling of the $t \bar{t}$ signal. Further progress is envisaged such that the precision could reach $500 \mathrm{MeV}$ [3] or even $200 \mathrm{MeV}$ [4] after the complete LHC programme. This standard approach is the subject of several other contributions in these proceedings $[5,6,7]$.

The challenges inherent in performing a per mil level quark mass measurement and in the interpretation of the measured mass within a field-theoretical mass scheme $[3,8]$ are addressed from several complementary angles:

- Estimates of non-perturbative effects and their uncertainties are continuously being refined and must evolve beyond the traditional comparison of several parton shower models.

- Dedicated studies are ongoing to understand the (hopefully universal) relation of the MC mass parameter with the pole mass $[9,10]$.

- Measurements that base the top quark measurement on alternative observables, with an orthogonal dependence on the main sources systematics, strengthen the world average.

- The top quark pole mass is extracted from a corrected measurement of the (differential) cross-section.

For a discussion of the first and second approaches, the reader is referred to other contributions to these proceedings [10].

Good examples of the third strategy are the methods which use the relation of the top quark mass with the B-hadron decay length [11] or the invariant mass of the $J / \psi$-lepton system [12, 13]. These methods, that are promising with large integrated luminosity, have been explored by the ATLAS and CMS experiments: Reference [14] determines the mass to a precision of $3 \mathrm{GeV}$ using the B-hadron decay length, while References [15] and [16] investigate the selection and reconstruction of $J / \psi$ in $t \bar{t}$ events. Two further methods that rely on relations of the top quark mass with certain features (peaks, end-points) of observables of the top quark decay products that 
have recently been deployed by the CMS experiment are presented in these proceedings: the top quark mass measurement from the $b$-jet energy peak and from the invariant mass $m_{b l}$ of the system formed by the $b$-jet and charged lepton from top quark decay.

The extraction of the top quark mass from the production cross-section forms an independent cross-check of the standard interpretation of the direct measurement in the pole mass scheme. Recent extractions of the top quark pole mass from the total cross-section are presented in Section 4, while the analysis of the differential $t \bar{t}+1$ jet cross-section is presented in Section 5.

\section{Top quark mass measurement from the b-jet energy peak}

In the rest frame of the top quark the energy of the b-quark produced in the $t \rightarrow W b$ decay has a simple relation with the top quark mass. In Reference [17] the authors show that under certain assumptions (in particular that of unpolarized production of the top quarks) the same relation holds for the peak position of the b-jet energy in the laboratory frame. Subsequent papers study how this observation can be used to measure the mass of new particles [18] and work generalize the study to multi-body decays [19].

CMS has applied this technique to the $8 \mathrm{TeV}$ proton-proton collision data collected in 2012 [20]. A very pure $t \bar{t}$ sample, with an expected $t \bar{t}$ contribution of $88 \%$ for the single b-tag and $95 \%$ for the double b-tag category, is selected in the di-lepton channel with an isolated electron and muon of opposite electric charge. The peak position in the $b$ - jet energy distribution is found with a fit to a $1 / E \log E$ function and calibrated to the parton-level b-quark energy, after which the top quark mass is found from the simple relation $m_{t}=E_{b}+\sqrt{m_{W}^{2}-m_{b}^{2}+E_{b}^{2}}$. The precision of the measurement is limited by uncertainties in the jet energy scale $(1.2 \mathrm{GeV})$ and in $t \bar{t}$ modelling $(2.1 \mathrm{GeV})$. The result, $m_{t}=172.3 \pm 2.9 \mathrm{GeV}$, is in excellent agreement with the world average.

\section{Template fit to the $m_{b l}$ distribution}

The second alternative mass determination I discuss measures the top quark mass from the distribution of the invariant mass of the system formed by the b-jet and the charged lepton. Following Ref. [21] the lepton and b-jet are combined that yield the minimal mass. This distribution is known to be invariant under Lorentz boosts. The extracted top quark mass is therefore expected to be insensitive to the production mechanism. The shape of the distribution depends on the top quark mass, with the most pronounced sensitivity around the end-point of the distribution (for $m_{l b}^{\min }=\sqrt{m_{t}^{2}-m_{W}^{2}}$.

In Ref. [22] the CMS collaboration reconstructs the $m_{l b}^{\min }$ distribution in the very pure electronmuon channel, using the full 2012 data set $\left(20 \mathrm{fb}^{-1}\right.$ at $\left.\sqrt{s}=8 \mathrm{TeV}\right)$. The top quark mass is determined by fitting the distribution to templates created with the MadGraph Monte Carlo generator. The mass is determined from the absolute and normalized distribution, with the shape analysis yielding more powerful results. The result is $m_{t}=172.3 \pm 1.3 \mathrm{GeV}$, with the dominant uncertainties in the modelling of top quark production and decay.

The mass extraction is repeated with templates based on a fixed-order prediction implemented in MCFM that describes top quark pair production at NLO and has leading-order accuracy for the decay. The parton-level distribution is "forward folded" to the detector-level (i.e. a MC estimate 
of the effects of hadronization and detector resolution is applied in the form of a smearing matrix and bin-by-bin efficiency correction). This is a promising step towards an extraction of the pole mass from the $m_{l b}^{\min }$ distribution. The relatively large shift of the result $(900 \mathrm{MeV}$, much larger than the scale uncertainty of $100 \mathrm{MeV}$ assigned to the MCFM prediction) demonstrates that for a pole mass extraction with reliable uncertainties the top quark decay must be incorporated at NLO in the calculation. Several authors [23] have pointed out that especially in the end-point region NLO decay and off-shell effects are sizeable.

\section{Extraction from the total $t \bar{t}$ production cross-section}

The classical alternative top mass measurement is the extraction of the mass from the total top quark pair production cross-section, first performed in Ref. [24, 25]. The mass is inferred by comparing the measured cross-section to a precise prediction of the dependence of the inclusive $t \bar{t}$ cross-section on the top quark mass. Among the advantages of this method is the possibility to unambiguously choose the renormalization scheme used in the calculation. This feature has been used to extract the running $\bar{M} S$ mass directly $[24,26]$. The theoretical uncertainty due to the truncation of the perturbative series is readily evaluated in the usual way, by varying the renormalization and factorization scales.

The top quark mass is extracted by maximizing the product of the likelihoods corresponding to the measured cross-section at center-of-mass energies of 7 and $8 \mathrm{TeV}$. Experimental and theoretical uncertainties are treated as nuisance parameters, taking into account the strong correlations between the 7 and $8 \mathrm{TeV}$ measurements. The most recent ATLAS and CMS measurements use the NNLO calculation of Ref. [27] with NNLL resummation to extract the pole mass, which reduces the scale uncertainty on the cross-section to the level of $3 \%$. An uncertainty of $1.7-1.8 \%$ is assigned to the cross-section to account for the uncertainty in the LHC beam energy.

The ATLAS analysis of Ref. [28] extracts the pole mass for $7 \mathrm{TeV}$ and $8 \mathrm{TeV}$ separately, and for three exponents of the previous generation of PDF sets (CT10, MSTW2008 and NNPDF2.1). The results obtained with different PDF sets are compatible with each other to within $200 \mathrm{MeV}$, but the results obtained on $7 \mathrm{TeV}$ and $8 \mathrm{TeV}$ data cluster around $171.4 \mathrm{GeV}$ and $174.1 \mathrm{GeV}$, respectively. Considering only uncorrelated experimental uncertainties, the two values are consistent at the level of 1.7 standard deviations. The combined fit yields $172.9_{-2.6}^{+2.5} \mathrm{GeV}$. The uncertainty is dominated by the PDF uncertainty, estimated as the full envelope of the error sets of the three PDFs, which amounts to $1.8 \mathrm{GeV}$.

The preliminary result of the cross-section measurement by CMS in the $e \mu$ channel analysis of the 7 and $8 \mathrm{TeV}$ data [29], presented in detail elsewhere in these proceedings [30], is slightly more precise than the ATLAS result [31,28]: where ATLAS finds an uncertainty of 3.9\% (4.3\%) at $\sqrt{s}=7 \mathrm{TeV}(\sqrt{s}=8 \mathrm{TeV})$ the CMS uncertainty is 3.6\% (3.9\%). Importantly, in both measurements the dependence of the measured cross-section on the assumed top quark mass is negligible ${ }^{1}$.

CMS uses the most recent PDF sets in the mass extraction, that include constraints from LHC data $^{2}$ and quotes several results that assume a given PDF set and $\alpha_{s}$ value. The mass values ex-

\footnotetext{
${ }^{1}$ In previous analyses [24, 26, 32] the straightforward interpretation of the extracted mass was somewhat obfuscated by this dependence.

${ }^{2}$ The MMHT14 and NNPDF3.0 sets include the LHC $t \bar{t}$ production cross-section measurements as a constraint.
} 
Table 1: The top quark pole mass extracted from the production cross-section.

\begin{tabular}{|c|c|c|c|c|}
\hline Experiment & pole mass & data & theory & comment \\
\hline D0 [25] & $169.1_{-5.2}^{+5.9} \mathrm{GeV}$ & $p \bar{p}, 1.96 \mathrm{TeV}, 1 \mathrm{fb}^{-1}$ & & \\
\hline $\begin{array}{l}\text { Langenfeld } \\
\text { et al. [24] }\end{array}$ & $168.9_{-3.4}^{+3.5} \mathrm{GeV}$ & idem & $\begin{array}{l}\text { through } \\
\bar{M} S \text { mass }\end{array}$ & \\
\hline D0 [26] & $167.5_{-4.9}^{+5.4} \mathrm{GeV}$ & $p \bar{p}, 1.96 \mathrm{TeV}, 5.3 \mathrm{fb}^{-1}$ & approx NNLO & \\
\hline CMS [32] & $176.7 \pm 2.9 \mathrm{GeV}$ & $p p, 7 \mathrm{TeV}, 5 \mathrm{fb}^{-1}$ & approx NNLO & \\
\hline ATLAS [28] & $172.9_{-2.6}^{+2.5} \mathrm{GeV}$ & $\begin{array}{l}p p, 7 \mathrm{TeV}, 5 \mathrm{fb}^{-1} \\
+8 \mathrm{TeV}, 20 \mathrm{fb}^{-1}\end{array}$ & NNLO+NNLL & $\begin{array}{c}\text { full PDF4LHC [34] } \\
\text { envelope of } 3 \text { PDF sets }\end{array}$ \\
\hline CMS [29] & $173.6_{-1.8}^{+1.7} \mathrm{GeV}$ & idem & idem & NNPDF3.0 [35], preliminary \\
\hline CMS [29] & $173.9_{-1.9}^{+1.8} \mathrm{GeV}$ & idem & idem & MMHT2014, preliminary \\
\hline CMS [29] & $174.1_{-2.2}^{+2.1} \mathrm{GeV}$ & idem & idem & CT14 [36], preliminary $\Omega$ \\
\hline ATLAS [37] & $173.7_{-2.1}^{+2.3} \mathrm{GeV}$ & $p p, 7 \mathrm{TeV}, 5 \mathrm{fb}^{-1}$ & $\mathrm{NLO} t \bar{t}+1$ jet & full PDF4LHC [34] \\
\hline
\end{tabular}

tracted from 7 and $8 \mathrm{TeV}$ data agree within $500 \mathrm{MeV}$. The values obtained with three different PDF sets span $500-600 \mathrm{MeV}$ and have not been combined into a single mass value ${ }^{3}$.

In Table 1 these results are compared to the ATLAS results and the other measurements of the top quark pole mass. The results are in good agreement with each other and with the world average from the direct measurements.

\section{Extraction from the differential $t \bar{t}+1$ jet cross-section}

A method proposed in Ref. [38] extracts the pole mass from the differential cross-section in top quark pair production in association with a hard jet $\left(d \sigma / d m_{t \bar{t} j}\right.$, with $m_{t \bar{t} j}$ the invariant mass of the system formed by the top quark pair and the extra jet). The enhanced sensitivity to the top quark mass avoids the limit on the precision of the quark pole mass extraction from the theory uncertainty on the inclusive $t \bar{t}$ production cross-section.

An ATLAS measurement [37] of the pole mass using this method on the $\sqrt{s}=7 \mathrm{TeV}$ data set collected in 2011 yields a result of $m_{t}^{\text {pole }}=173.7 \pm 1.5$ (stat.) \pm 1.4 (syst.) ${ }_{-0.5}^{+1.0} \mathrm{GeV}$, where the latter term represents the theory uncertainty, estimated from variations of the renormalization and factorization scales and the envelope of the error sets from PDF fits.

\section{Summary and prospects}

The key goal of alternative top quark mass measurement is to provide an independent confirmation of the interpretation of the more precise standard method. With the publication of the results of the pole mass extraction from the inclusive top quark pair production cross-section measured at

Even if the bias on the mass due to the circularity of the exercise is expected to be negligible at present, an effort must be made to improve the PDFs without sacrificing a key observable in this mass determination and many constraints on physics beyond the Standard Model.

${ }^{3}$ The PDF4LHC recommendation [33] for run II explicitly requests top quark mass extraction using individual PDF sets, as provided by both collaborations. The new recommendation for obtaining the total PDF uncertainty using the combined PDF set is less conservative than the envelope. 
7 and $8 \mathrm{TeV}[28,29]$ and from the differential $t \bar{t}+1$ jet cross-section at $7 \mathrm{TeV}$ [37] the precision of such measurements approaches $1 \%$. To that level of precision the results are in excellent agreement with the world average.

Further progress can be made by combining the ATLAS and CMS measurements, by reducing systematics on the $13 \mathrm{TeV}$ data and by improving the PDF fit by including LHC data. A significant reduction of the weight of the PDF uncertainty is expected at $\sqrt{s}=13 \mathrm{TeV}$, where top quark pair production requires a smaller fraction $x$ of the proton momentum. If both the experimental and PDF uncertainties are reduced considerably, the precision of the mass extraction is limited by the scale uncertainty of the NNLO+NNLL calculation ${ }^{4}$ to approximately $1 \mathrm{GeV}$.

The extraction from the differential cross-section [37] can improve considerably by including the $8 \mathrm{TeV}$ (and $13 \mathrm{TeV}$ ) data. With a finer-grained binning the sensitivity to the top quark mass increases considerably. The authors of Ref. [38] expect a $1 \mathrm{GeV}$ precision can be achieved with the data set collected in 2012 and 2015.

Methods like the $m_{b l}$ analysis performed by CMS [22] have shown good sensitivity to the top quark mass. With a more sophisticated (NLO) treatment of the modeling of the top quark decay and a careful evaluation of the MC mass dependence of the unfolding and of the theory uncertainty such measurements can form valuable pole mass measurements.

\section{References}

[1] ATLAS, CDF, CMS, D0 Collaboration, First combination of Tevatron and LHC measurements of the top-quark mass, arXiv:1403.4427.

[2] M. Beneke et al., Top quark physics, in 1999 CERN Workshop on standard model physics (and more) at the LHC, CERN, Geneva, Switzerland, 25-26 May: Proceedings, 2000. hep-ph/0003033.

[3] A. Juste, S. Mantry, A. Mitov, A. Penin, P. Skands, E. Varnes, M. Vos, and S. Wimpenny, Determination of the top quark mass circa 2013: methods, subtleties, perspectives, Eur. Phys. J. C74 (2014), no. 103119 , [arXiv:1310.0799].

[4] CMS Collaboration, Projected improvement of the accuracy of top-quark mass measurements at the upgraded LHC, CMS-PAS-FTR-13-017.

[5]

[6] C. Deterre, Top-quark mass measurements at the tevatron (incl. tevatron combination), these proceedings (2015).

[7] A. Maier, Statistical and systematic treatment issues in top mass combinations, these proceedings (2015).

[8] S. Moch et al., High precision fundamental constants at the TeV scale, arXiv:1405.4781.

[9] A. H. Hoang, The Top Mass: Interpretation and Theoretical Uncertainties, arXiv:1412.3649.

[11] C. S. Hill, J. R. Incandela, and J. M. Lamb, A Method for measurement of the top quark mass using the

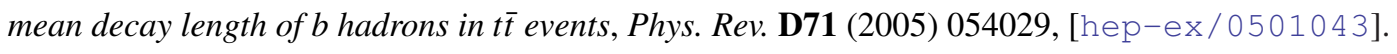

\footnotetext{
${ }^{4}$ The scale variation of the NNLO calculation in the $\bar{M} S$ scheme is about half that of the NNLO+NNLL calculation in the pole mass scheme, but the consensus is that in this case the uncertainty may be underestimated.
} 
[12] A. Kharchilava, Top mass determination in leptonic final states with J/ $\psi$, Phys. Lett. B476 (2000) 73-78, [hep-ph/9912320].

[13] R. Chierici and A. Dierlamm, Determination of the top mass with exclusive events $t->W b->l v J / \psi$ $X$, Tech. Rep. CMS-NOTE-2006-058, CERN, Geneva, May, 2006.

[14] CMS Collaboration, Measurement of the top quark mass using the B-hadron lifetime technique, CMS-PAS-TOP-12-030.

[15] ATLAS Collaboration, Reconstruction of $J / \psi$ mesons in $t \bar{t}$ final states in proton-proton collisions at $\sqrt{s}=8 \mathrm{TeV}$ with the ATLAS detector, ATLAS-CONF-2015-040.

[16] CMS Collaboration, Study of the underlying event, $b$-quark fragmentation and hadronization

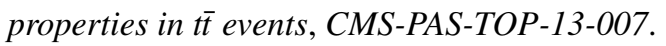

[17] K. Agashe, R. Franceschini, and D. Kim, Simple 'invariance' of two-body decay kinematics, Phys. Rev. D88 (2013), no. 5 057701, [arXiv:1209.0772].

[18] K. Agashe, R. Franceschini, and D. Kim, Using Energy Peaks to Measure New Particle Masses, JHEP 11 (2014) 059, [arXiv:1309.4776].

[19] K. Agashe, R. Franceschini, D. Kim, and K. Wardlow, Mass Measurement Using Energy Spectra in Three-body Decays, arXiv:1503.03836.

[20] CMS Collaboration, Measurement of the top quark mass from the b jet energy spectrum, CMS-PAS-TOP-15-002.

[21] S. Biswas, K. Melnikov, and M. Schulze, Next-to-leading order QCD effects and the top quark mass measurements at the LHC, JHEP 08 (2010) 048, [arXiv: 1006.0910 ].

[22] CMS Collaboration, Determination of the top-quark mass from the $m(l b)$ distribution in dileptonic ttbar events at $\operatorname{sqrt}(s)=8 \mathrm{TeV}, \mathrm{CMS}-\mathrm{PAS}-\mathrm{TOP}-14-014$.

[23] G. Heinrich, A. Maier, R. Nisius, J. Schlenk, and J. Winter, NLO QCD corrections to $W^{+} W^{-} b \bar{b}$ production with leptonic decays in the light of top quark mass and asymmetry measurements, JHEP 06 (2014) 158, [arXiv:1312.6659].

[24] U. Langenfeld, S. Moch, and P. Uwer, Measuring the running top-quark mass, Phys.Rev. D80 (2009) 054009, [arXiv:0906.5273].

[25] D0 Collaboration, V. M. Abazov et al., Combination of t anti-t cross section measurements and constraints on the mass of the top quark and its decays into charged Higgs bosons, Phys. Rev. D80 (2009) 071102, [arXiv:0903.5525].

[26] D0 Collaboration, V. M. Abazov et al., Determination of the pole and MSbar masses of the top quark from the t $\bar{t}$ cross section, Phys. Lett. B703 (2011) 422-427, [arXiv:1104.2887].

[27] M. Czakon, P. Fiedler, and A. Mitov, Total Top-Quark Pair-Production Cross Section at Hadron Colliders Through $O\left(\alpha_{s}^{4}\right)$, Phys. Rev. Lett. 110 (2013), no. 25 252004, [arXiv: 1303.6254 ].

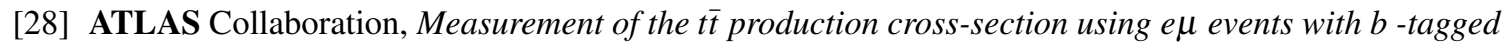
jets in pp collisions at $\sqrt{s}=7$ and 8 TeV with the ATLAS detector, Eur. Phys. J. C74 (2014), no. 10 3109, [arXiv:1406.5375].

[29] CMS Collaboration, Measurement of the $t \bar{t}$ production cross section in the e $\mu$ channel in pp collisions at 7 and $8 \mathrm{TeV}, \mathrm{CMS}-\mathrm{PAS}-\mathrm{TOP}-13-004$.

[30] S. Crucy, Inclusive top pair production at 7, 8 and 13 tev in cms, these proceedings (2015). 
[31] J. Glatzer, Inclusive top pair production at 7, 8 and 13 tev in atlas, these proceedings (2015).

[32] CMS Collaboration, Determination of the top-quark pole mass and strong coupling constant from the $t \bar{t}$ production cross section in pp collisions at $\sqrt{s}=7 \mathrm{TeV}$, Phys. Lett. B728 (2014) 496-517, [arXiv:1307.1907]. [Erratum: Phys. Lett.B728,526(2014)].

[33] J. Butterworth et al., PDF4LHC recommendations for LHC Run II, arXiv : 1510.03865.

[34] J. Rojo et al., The PDF4LHC report on PDFs and LHC data: Results from Run I and preparation for Run II, J. Phys. G42 (2015) 103103, [arXiv: 1507.00556 ].

[35] NNPDF Collaboration, R. D. Ball et al., Parton distributions for the LHC Run II, JHEP 04 (2015) 040, [arXiv:1410.8849].

[36] S. Dulat et al., The CT14 Global Analysis of Quantum Chromodynamics, arXiv: 1506.07443.

[37] ATLAS Collaboration, Determination of the top-quark pole mass using $t \bar{t}+1$-jet events collected with the ATLAS experiment in 7 TeV pp collisions, JHEP 10 (2015) 121, [arXiv: 1507.01769 ].

[38] S. Alioli et al., A new observable to measure the top-quark mass at hadron colliders, Eur. Phys. J. C73 (2013) 2438, [arXiv:1303.6415]. 